\title{
QUADRATIC ZERO-ONE LAWS FOR GAUSSIAN MEASURES AND THE DISTRIBUTION OF QUADRATIC FORMS
}

\author{
ALEJANDRO de ACOSTA
}

ABSTRACT. Given a centered Gaussian measure on a vector space, the set of points where a sequence of quadratic forms converges to a constant has probability zero or one. The distribution of a quadratic form under a Gaussian measure is either point mass at zero or absolutely continuous.

1. Introduction. Limit theorems for sequences of quadratic functionals of Gaussian processes are well known in probability theory. Let us mention two examples:

(1) Let $\left\{B_{t}\right\}, t \in[0,1]$, be a standard Brownian motion. Then a classical theorem of P. Lévy asserts

$$
P\left[\lim _{n} \sum_{j=1}^{2^{n}}\left[B\left(j / 2^{n}\right)-B\left((j-1) / 2^{n}\right)\right]^{2}=1\right]=1 .
$$

G. Baxter and other authors have extended this result to a broad class of Gaussian processes (see [6, p. 88, and references therein]).

(2) Let $\left\{X_{n}\right\}, n \in N$, be a sequence of independent random variables with common distribution $N(0,1)$. By the strong law of large numbers,

$$
P\left[\lim _{n} \frac{1}{n} \sum_{j=1}^{n} X_{j}^{2}=1\right]=1 \text {. }
$$

In this work-\$3-we prove that given a centered Gaussian measure on a vector space, the set of points where a sequence of quadratic forms (of arbitrary sign) converges to a constant has probability zero or one. This result may be regarded as a general zero-one dichotomy underlying limit theorems of the above types.

Examples (1) and (2) are significant cases in which the set of convergence of a sequence of quadratic forms to a nonzero constant has measure one. In $\S 4$ we prove that for a nonzero constant the alternative of measure one is impossible when the sequence reduces to a single quadratic form. In fact, we prove a stronger result: the distribution of a quadratic form under a centered Gaussian measure is either point mass at zero or absolutely continuous with respect to Lebesgue measure (see Theorem 4.1 for more detailed information). For the particular case when the Gaussian measure is on a separable Hilbert space and the quadratic form is the square of the norm, the result has been obtained by Kuelbs and Kurtz [9].

Received by the editors February 21, 1975.

AMS (MOS) subject classifications (1970). Primary 60B05, 60F20, 60G15, 28A40.

( American Mathematical Society 1976 
2. Preliminaries on Gaussian measures. In this section we collect some definitions and results on Gaussian measures. We adopt the point of view presented in Badrikian and Chevet [3, p. 364ff]; it is based on Fernique's [8] generalization of a classical characterization of centered Gaussian distributions on the real line due to Bernstein.

A measurable vector space is a pair ( $\left.E, 9 \frac{1}{6}\right)$, with $E$ a real vector space and 93 a $\sigma$-algebra of subsets of $E$ such that:

(a) The map $(x, y) \rightarrow x+y$ from $E \times E$ into $E$ is $\mathscr{B} \otimes \mathscr{B}-\mathscr{B}$ measurable.

(b) The map $(\lambda, x) \rightarrow \lambda x$ from $R \times E$ into $E$ is $\mathscr{R} \otimes \mathscr{B}-\mathscr{G}$ measurable, where $\mathcal{R}$ is the Borel $\sigma$-algebra on $R$.

For each $\theta \in R$, let $T_{\theta}: E \times E \rightarrow E \times E$ be defined by

$$
T_{\theta}((x, y))=(x \cos \theta+y \sin \theta, x \sin \theta-y \cos \theta) .
$$

Then $T_{\theta}$ is $\mathscr{\Re} \mathscr{B}-\mathscr{B} \otimes \mathscr{T}$ measurable for each $\theta \in R$.

A probability measure (p.m.) $\mu$ on $(E, \mathscr{T})$ will be called $B$-Gaussian (Gaussian in the sense of Bernstein) if

$$
(\mu \otimes \mu) \circ T_{\theta}^{-1}=\mu \otimes \mu \text { for all } \theta \in R .
$$

In order to clarify the connection between this definition and the usual definition of a Gaussian measure, we consider two situations:

(1) Given a dual system of vector spaces $(E, F)$, let $9(E, F)$ be the $\sigma$-algebra induced by $F$ on $E$; then $(E, \quad h(E, F))$ is a measurable vector space. A p.m. $\mu$ on $\mathscr{B}(E, F)$ is centered Gaussian if $\mu \circ f^{-1}$ is centered Gaussian in $R$ for all $f \in F$. It can be proved that a p.m. $\mu$ on $\mathscr{B}(E, F)$ is $B$-Gaussian if and only if it is centered Gaussian.

(2) Let $E$ be a real locally convex (Hausdorff) topological vector space, $E^{\prime}$ its topological dual space. Let $Q$ be the Borel $\sigma$-algebra of $E$. Then $(E, Q)$ is not, in general, a measurable vector space. However, if $\mathscr{F}$ is the Borel $\sigma$-algebra of $E \times E$, then the maps $T_{\theta}$ are $\mathscr{F}-\mathscr{F}$ measurable, since they are continuous for the product topology. It is known that if $\nu$ is a regular p.m. on $(E, Q)$, then the product measure $\nu \otimes \nu$ on $Q \otimes Q$ has a unique regular extension $\overline{\nu \otimes \nu}$ to $\mathcal{F}$ (see [2, p. 92]). Let $\mu$ be a regular p.m. on $(E, \mathbb{Q})$ which is centered Gaussian relative to the duality $\left(E, E^{\prime}\right)$. Then it can be shown that $\mu$ satisfies [3, p. 366]

$$
(\overline{\mu \otimes \mu}) \circ T_{\theta}^{-1}=\overline{\mu \otimes \mu} \quad \text { for all } \theta \in R .
$$

It may be proved [3, p. 372] that the support of $\mu$,

$$
S(\mu)=\{x \in E: \mu(U)>0 \text { for every open set } U \text { containing } x\},
$$

is a closed subspace with $\mu(S(\mu))=1$; further, if $\Phi$ is the covariance form of $\mu$,

$$
\Phi(y, z)=\int\langle x, y\rangle\langle x, z\rangle \mu(d x), \quad y, z \in E^{\prime},
$$

and $N=\{y: \Phi(y, y)=0\}$, then $S(\mu)=N^{\perp}$ and $[S(\mu)]^{\perp}=N$.

\section{Bilinear and quadratic zero-one laws.}

TheOREM 3.1. Let (E, $\cap)$ be a measurable vector space. Let $\mu$ be a $B$ Gaussian p.m. on $\left(E, \Re^{\prime}\right)$, and let $\Re_{\mu}$ be the $\mu$-completion of $\Re^{\prime}$. Let $\left\{A_{n}\right\}$, $n \in N$, be a sequence of $\mathscr{G}_{\mu} \otimes \mathscr{h}_{\mu}$-measurable bilinear forms on $E \times E$. Then 


$$
(\mu \otimes \mu)\left\{(x, y): \lim _{n} A_{n}(x, y)=0\right\}=0 \quad \text { or } 1 .
$$

Proof. For each $y \in E$, let $S(y)=\left\{x: \lim _{n} A_{n}(x, y)=0\right\}$. Then $S(y)$ is a subspace and $S(y) \in \mathscr{B}_{\mu}$. By the zero-one law for subspaces (see [3, p. 367]), we have $\mu(S(y))=0$ or 1 .

Let $G=\{y: \mu(S(y))=1\}$. Then $G \in \Re_{\mu}$. We claim that $G$ is a subspace. In fact, $S(\lambda y)=S(y)$ if $\lambda \neq 0$ and $S(\lambda y)=E$ if $\lambda=0$; also $S(y+z)$ $\supset S(y) \cap S(z)$. It follows that $\mu(S(y))=1$ implies $\mu(S(\lambda y))=1$ for all $\lambda \in R$ and $\mu(S(y))=1$ and $\mu(S(z))=1$ imply $\mu(S(y+z))=1$.

We have now

$$
\begin{gathered}
(\mu \otimes \mu)\left\{(x, y): \lim _{n} A_{n}(x, y)=0\right\}=\int_{E} \mu\left\{x: \lim _{n} A_{n}(x, y)=0\right\} \mu(d y) \\
=\int_{E} \mu(S(y)) \mu(d y)=\int_{G} 1 \cdot d \mu=\mu(G)=0 \text { or } 1 . \square
\end{gathered}
$$

REMARKS. (1) Theorem 3.1 may be extended with the same proof to stable measures under the (slightly more restrictive) hypotheses of the DudleyKanter [7] zero-one laws for subspaces under stable measures.

(2) Theorem 3.1 is true, with the same method of proof, for a sequence of multilinear forms.

(3) Theorem 3.1 remains valid for a triple $(E, Q, \mu)$ as described in (2) of $\S 2$. In fact, the zero-one law is still true for $\Theta_{\mu}$-measurable subspaces (see $[3, \mathrm{p}$. 367]).

TheOREM 3.2. Let (E, 9 ) be a measurable vector space. Let $\mu$ be a $B$ Gaussian p.m. on $\left(E, \Re^{3}\right)$, and let $\Re_{\mu}$ be the $\mu$-completion of $\Re_{3}$. Let $\left\{A_{n}\right\}$, $n \in N$, be a sequence of $\mathscr{G}_{\mu} \otimes \mathscr{G}_{\mu}$-measurable symmetric bilinear forms on $E \times E$. Then for all $c \in R, \mu\left\{x: \lim _{n} A_{n}(x, x)=c\right\}=0$ or 1 .

Proof. Let $B=\left\{(x, y): \lim _{n}\left[A_{n}(x, x)-A_{n}(y, y)\right]=0\right\}$. We claim that $(\mu \otimes \mu)(B)=0$ or 1 . In fact, let $C=\left\{(x, y): \lim _{n} A_{n}(x, y)=0\right\}$, and let $T$ : $E \times E \rightarrow E \times E$ be defined by

$$
T((x, y))=\left(2^{-1 / 2}(x+y), 2^{-1 / 2}(x-y)\right) .
$$

Since $A_{n}(x, x)-A_{n}(y, y)=2 A_{n}\left(2^{-1 / 2}(x+y), 2^{-1 / 2}(x-y)\right)$, we have

$$
(\mu \otimes \mu)(B)=(\mu \otimes \mu)\left(T^{-1}(C)\right) \text {. }
$$

Since $\mu$ is $B$-Gaussian,

$$
(\mu \otimes \mu) \circ T^{-1}(C)=(\mu \otimes \mu)(C)=0 \text { or } 1 \quad \text { by Theorem } 3.1 .
$$

Now let $D=\left\{x: \lim _{n} A_{n}(x, x)=c\right\}$, and observe that $D \times D=(D \times$ $E) \cap B$. We have

$$
\begin{aligned}
(\mu(D))^{2} & =(\mu \otimes \mu)(D \times D)=(\mu \otimes \mu)((D \times E) \cap B) \\
& =0 \quad \text { if }(\mu \otimes \mu)(B)=0 \\
& =\mu(D) \quad \text { if }(\mu \otimes \mu)(B)=1 .
\end{aligned}
$$

Remark. Theorem 3.2 remains valid for a triple $\left(E, Q^{\prime}, \mu\right)$ as described in (2) of $\$ 2$. In fact, in this case the proof goes through by using the second version 
of Theorem 3.1 (see Remark (3) following it) and the fact that $(\overline{\mu \otimes \mu}) \circ T^{-1}$ $=\overline{\mu \otimes \mu}$.

4. The distribution of quadratic forms. We recall some definitions which will be used in Theorem 4.1. Let $E$ be a vector space, $E^{*}$ its algebraic dual space. Let $A$ be a symmetric bilinear form on $E \times E$, and let $\alpha_{A}: E \rightarrow E^{*}$ be the linear map defined by $\left\langle x, \alpha_{A}(y)\right\rangle=A(x, y)$ for $x, y \in E$. The kernel of $A$, denoted $\operatorname{Ker} A$, is the subspace $\alpha_{A}^{-1}(\{0\})$. Given a subspace $S$ of $E$, the restriction of $A$ to $S \times S$ will be denoted $A_{S}$.

Let $E$ be bornological locally convex topological vector space (see, e.g., [10]). It is known that if $u$ is a sequentially continuous linear form on $E$, then $u \in E^{\prime}$ (see [5]). This is the reason for introducing the assumption that $E$ is bornological in the following proposition.

THEOREM 4.1. Let $E$ be a real bornological locally convex topological vector

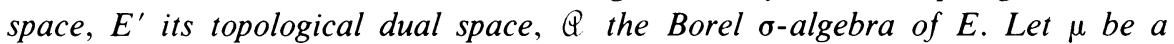
regular centered Gaussian measure on $\left(E, \hat{A}^{\prime}\right)$, let $S$ be the support of $\mu$, and let $Q_{\mu}$ be the $\mu$-completion of $Q$.

Let $A$ be a symmetric bilinear form on $E \times E$ such that (a) $A$ is $\mathbb{A}_{\mu} \otimes \mathbb{Q}_{\mu}$ measurable and (b) $\alpha_{A}(E) \subset E^{\prime}$. Let $d=\operatorname{codim}_{S} \operatorname{Ker} A_{S}$.

Let $f(x)=A(x, x)$ for $x \in E$, and let $\nu=\mu \circ f^{-1}$. Then

if $d=0$, then $\nu=\delta_{0}$;

if $d \geqslant 1$, then $\nu$ is absolutely continuous with respect to Lebesgue measure;

if $d \geqslant 3$, then $\nu$ has a bounded uniformly continuous density with respect to Lebesgue measure.

We need some lemmas. The first two are concerned with some auxiliary results of an algebraic and algebraic-topological nature. The third one is quite essential.

LeMmA 4.1. Let $E$ be a vector space, $E^{*}$ its algebraic dual space, $G$ a subspace of $E^{*}$. Let $\Phi$ be a symmetric positive semidefinite bilinear form on $G \times G$, and let $N=\{v: \Phi(v, v)=0\}$.

Let $\alpha: E \rightarrow G$ be a linear map. Define a linear map $\beta: E \rightarrow E^{*}$ by $<x$, $\beta(y)\rangle=\Phi(\alpha(x), \alpha(y))$ for $x, y \in E$. Then

$$
\operatorname{codim}_{N^{\perp}} N^{\perp} \cap \alpha^{-1}(N) \leqslant \operatorname{codim}_{\beta(E)} \beta(E) \cap N .
$$

Proof. By the positive semidefinite character of $\Phi, N$ is a subspace. Using again this property of $\Phi$, it is easily shown that $\beta^{-1}(\{0\})=\alpha^{-1}(N)$.

In order to prove the inequality, it is enough to show: if $T$ is a subspace of $N^{\perp}$ such that $T \cap \alpha^{-1}(N)=\{0\}$, then

(1) $\beta$ is one-to-one on $T$,

(2) $\beta(T) \cap N=\{0\}$.

Statement (1) is clear since $T \cap \beta^{-1}(\{0\})=\{0\}$. To prove (2), suppose $z \in T$ and $\beta(z) \in N$. Then $\Phi(\alpha(z), \alpha(z))=\langle z, \beta(z)\rangle=0$, and $z \in \alpha^{-1}(N)$. But this implies $z=0$.

LEMMA 4.2. Let $E$ be a bornological locally convex topological vector space, $E^{\prime}$ its topological dual space. Let $\Phi$ be a symmetric positive semidefinite bilinear 
form on $E^{\prime} \times E^{\prime}$ such that the map $u \rightarrow \Phi(u, u)$ is sequentially $\sigma\left(E^{\prime}, E\right)$ continuous.

Let $A$ be a symmetric bilinear form on $E \times E$, such that $\alpha_{A}(E) \subset E^{\prime}$. Let $\beta: E \rightarrow E^{*}$ be the linear map defined by $\langle x, \beta(y)\rangle=\Phi\left(\alpha_{A}(x), \alpha_{A}(y)\right)$. Then $\beta(E) \subset E^{\prime}$.

Proof. Let us show first that $\alpha_{A}$ is continuous from $E$ into $E^{\prime}$ endowed with $\sigma\left(E^{\prime}, E\right)$. In fact, if $\left\{x_{i}\right\}$ is a net in $E, x_{i} \rightarrow 0$, then for all $y \in E$,

$$
\left\langle y, \alpha_{A}\left(x_{i}\right)\right\rangle=A\left(y, x_{i}\right)=A\left(x_{i}, y\right)=\left\langle x_{i}, \alpha_{A}(y)\right\rangle \rightarrow 0
$$

because $\alpha_{A}(y) \in E^{\prime}$.

Next we observe that for each $v \in E^{\prime}$, the map $u \rightarrow \Phi(u, v)$ is a sequentially $\sigma\left(E^{\prime}, E\right)$-continuous linear form on $E^{\prime}$.

Let $y \in E$; since $\langle\cdot, \beta(y)\rangle=\Phi\left(\alpha_{A}(\cdot), \alpha_{A}(y)\right)$, it follows that $\beta(y)$ is a sequentially continuous linear form on $E$. The assumption that $E$ is bornological implies that $\beta(y) \in E^{\prime}$.

Lemma 4.3. Assume that the hypotheses of Theorem 4.1 are true, omitting the bornological condition. Let

$$
g(x)=\int_{E}(A(x, z))^{2} \mu(d z) \text { for } x \in E .
$$

Then

(a) $|\hat{v}(t)|^{2}=\int_{E} \exp \left(-2 t^{2} g\right) d \mu$,

(b) Let $r>0$. Then $\int_{E} g^{-r} d \mu<\infty$ implies $|t|^{r}|\hat{v}(t)| \rightarrow 0$ as $|t| \rightarrow \infty$.

Proof. Let $T: E \times E \rightarrow E \times E$ be defined by $T((x, y))=\left(2^{-1 / 2}(x+y)\right.$, $\left.2^{-1 / 2}(x-y)\right)$. Then

$$
\begin{aligned}
|\hat{v}(t)|^{2} & =\hat{v}(t) \overline{\hat{v}(t)}=\left(\int \exp (\text { itf }) d \mu\right)\left(\overline{\int \exp (i t f) d \mu}\right) \\
& =\iint \exp (i t[A(x, x)-A(x, y)]) d \mu(x) d \mu(y) \\
& =\iint \exp (i 2 t(A \circ T)) d(\mu \otimes \mu),
\end{aligned}
$$

because $2 A\left(2^{-1 / 2}(x+y), 2^{-1 / 2}(x-y)\right)=[A(x, x)-A(y, y)]$.

By the Gaussian character of $\mu$ (see (2) of $\S 2$ ),

$$
\begin{aligned}
\iint \exp (12 t(A \circ T)) d(\mu \otimes \mu) & =\iint \exp (i 2 t A) d(\overline{\mu \otimes \mu}) \circ T^{-1} \\
& =\iint \exp (i 2 t A) d(\mu \otimes \mu) \\
& =\int \mu(d x) \int \exp (i 2 t A(x, y)) \mu(d y) \\
& =\int \mu(d x) \exp \left(-2 t^{2} g(x)\right)
\end{aligned}
$$

since $A(x, \cdot)$ is a Gaussian random variable on $(E, Q, \mu)$ with variance $g(x)$. This proves (a).

An elementary calculation shows: for any $a>0$,

$$
\max _{t \in R}|t|^{2 r} \exp \left(-a t^{2}\right)=r^{r} \exp (-r) a^{-r} \text {. }
$$


Let $h_{t}(x)=|t|^{2 r} \exp \left(-2 t^{2} g(x)\right)$. Then

(I) if $g(x)>0$, then $\lim _{t \rightarrow \infty} h_{t}(x)=0$,

(II) $\left|h_{t}(x)\right| \leqslant r^{r} \exp (-r)(2 g(x))^{-r}$ for all $t \in R, x \in E$.

Assume now $\int g^{-r} d \mu<\infty$; then $\mu\{x: g(x)>0\}=1$. By (I), (II) and the Lebesgue dominated convergence theorem, we have

$$
\lim _{t \rightarrow \infty}|t|^{r}|\hat{v}(t)|=\lim _{t \rightarrow \infty}\left(\int h_{t} d \mu\right)^{1 / 2}=\left(\int\left(\lim _{t \rightarrow \infty} h_{t}\right) d \mu\right)^{1 / 2}=0 .
$$

Proof of Theorem 4.1. Let us remark first that $f$ is $\mathbb{Q}_{\mu}$-measurable; this follows at once from property (a) of $A$.

If $\operatorname{Ker} A_{S}=S$, then $f^{-1}(\{0\}) \supset S$; therefore $\nu(\{0\})=1$. Now suppose Ker $A_{S} \neq S$; then $d=\operatorname{codim}_{S} \operatorname{Ker} A_{S} \geqslant 1$.

We will consider three cases: (a) $d \geqslant 3$, (b) $d=2$, (c) $d=1$.

(a) Assume $d \geqslant 3$. Let $\Phi$ be the covariance form of $\mu$ and $N=\{u$ : $\Phi(u, u)=0\}$. Recalling that $S=N^{\perp}$ and $S^{\perp}=N$, it is easily verified that

$$
\operatorname{Ker} A_{S}=N^{\perp} \cap \alpha_{A}^{-1}(N) \text {. }
$$

Let $\beta: E \rightarrow E^{*}$ be the linear map defined by

$$
\langle x, \beta(y)\rangle=\int A(z, x) A(z, y) \mu(d z)=\Phi\left(\alpha_{A}(x), \alpha_{A}(y)\right) .
$$

Since $\hat{\mu}(u)=\exp \left(-\frac{1}{2} \Phi(u, u)\right)$ and characteristic functionals are sequentially $\sigma\left(E^{\prime}, E\right)$-continuous, it follows that $u \rightarrow \Phi(u, u)$ is sequentially $\sigma\left(E^{\prime}, E\right)$ continuous. Since by assumption $\alpha_{A}(E) \subset E^{\prime}$, Lemma 4.2 implies that $\beta(E)$ $\subset E^{\prime}$.

By (*) and Lemma 4.1, we have $\operatorname{codim}_{\beta(E)} \beta(E) \cap N \geqslant d \geqslant 3$. Now every element of $\beta(E)$ is an element of $E^{\prime}$ continuous with respect to the seminorm $x \rightarrow(g(x))^{1 / 2}$, where $g(x)=\langle x, \beta(x)\rangle$. Applying Theorem 4.1 of [1] (the hypotheses stated there differ somewhat from those of the present theorem, but the proof goes through without changes), we obtain the result

$$
\int g^{-r / 2} d \mu<\infty \text { for any } r<3 \text {. }
$$

By Lemma 4.3, this implies $\int|\hat{v}(t)|^{2} d t<\infty$. From another result of Fourier analysis (see, e.g., [4, p. 147]) it follows that $\nu$ is absolutely continuous with respect to Lebesgue measure.

(b) If $d=2$, we may argue as in (a) to obtain

$$
\int g^{-p / 2} d \mu<\infty \text { for any } p<2 \text {. }
$$

By Lemma 4.3, this implies $\int|\hat{v}(t)|^{2} d t<\infty$. From another result of Fourier analysis (see, e.g., [4, p. 147], it follows that $\nu$ is absolutely continuous with respect to Lebesgue measure.

(c) Let $d=1$. Since $\operatorname{Ker} A_{S}=\bigcap_{y \in S}\{x \in S: A(x, y)=0\}$, it follows that $V=\operatorname{Ker} A_{S}$ is a closed subspace and the canonical projection $\pi: S \rightarrow S / V$ is a continuous linear map from $S$ onto the one-dimensional space $S / V$.

Let $f_{S}=f \mid S$. We claim that $f_{S}(x)=f_{S}(y)$ whenever $x-y \in V$. In fact, $A_{S}(x, x)-A_{S}(y, y)=A_{S}(x+y, x-y)=0$. It follows that there exists a function $\tilde{f}$ on $S / V$ such that $f_{S}=\tilde{f} \circ \pi$. Clearly $\tilde{f}$ is a quadratic form on $S / V$. 
Let $\mu_{S}=\mu \mid S, \tilde{\mu}=\mu_{S} \circ \pi^{-1}$. Then $\tilde{\mu}$ is a centered Gaussian measure on $S / V$; since $\tilde{\mu}(\{0\})=\mu_{S}(V)=0$ (because $V$ is a closed subspace properly contained in $S$ ), the measure $\tilde{\mu}$ is nondegenerate.

It is an elementary fact that $\tilde{\mu} \circ \tilde{f}^{-1}$ is absolutely continuous with respect to Lebesgue measure. But

$$
\mu \circ f^{-1}=\mu_{S} \circ f_{S}^{-1}=\mu_{S} \circ(\tilde{f} \circ \pi)^{-1}=\tilde{\mu} \circ \tilde{f}^{-1} .
$$

REMARK. If $d=1$, then the density of $\nu$ does not have a bounded uniformly continuous version; in fact, $\nu$ is-except for a scale factor-the chi-square distribution. If $d=2$, then the density of $\nu$ may fail to possess a version with the stated properties. For example, there is no bounded version of the density of the distribution of the quadratic form $f((u, v))=u^{2}-v^{2}$ under the standard normal distribution in $R^{2}$.

\section{REFERENCES}

1. A. de Acosta, (1975) Stable measures and seminorms, Ann. Probability 3.

2. A. Badrikian, (1970) Séminaire sur les fonctions aléatoires linéaires et les mesures cylindriques, Lecture Notes in Math., vol. 139, Springer-Verlag, Berlin and New York. MR43 \#4994.

3. A. Badrikian and S. Chevet, (1974) Measures cylindriques, espaces de Wiener et fonctions aléatoires Gaussiens, Lecture Notes in Math., vol. 379, Springer-Verlag, Berlin and New York.

4. K. L. Chung, (1968) A course in probability theory, Harcourt, Brace \& World, New York. MR37 \#4842.

5. R. M. Dudley, (1964) On sequential convergence, Trans. Amer. Math. Soc. 112, 483-507; Corrections, ibid. 148(1970), 623-624. MR30 \# 5266; 41, 2608.

6. , (1973) Sample functions of the Gaussian process, Ann. Probability 1, 66-103.

7. R. M. Dudley and M. Kanter, (1974) Zero-one laws for stable measures, Proc. Amer. Math. Soc. 45, 245-252.

8. X. Fernique, (1971) Régularité des processus gaussiens, Invent. Math. 12, 304-320. MR44 \#3380.

9. J. D. Kuelbs and T. Kurtz, (1974) Berry-Esséen estimates in Hilbert space and an application to the law of the iterated logarithm, Ann. Probability 2, 387-407.

10. H. H. Schaefer, (1966) Topological vector spaces, Macmillan, New York and London. MR33 \# 1689.

Departmento de Matématica, Universidad Nacional de la Plata, la Plata, Argentina

Current address: Departamento de Matemáticas, Instituto Venezolano de Investigaciones Científicas, Apartado 1827, Caracas, Venezuela 